\title{
Some properties and a characterization of two-directional scaling function
}

\author{
Wan-She Li , Ting-Ting Hu \\ Mathematics and information Science of shaan xi Normal University, Xian, China \\ liwsh@snnu.edu.cn; htt880420@126.com
}

Keywords: two direction refinable equation; two direction MRA two direction scaling function; the Parseval frame.

Abstract. In this paper, we denote two direction MRA and two direction scaling function in $L^{2}(R)$ with dilation factor 2 , discuss the proposition of two direction scaling function, give a necessary and sufficient condition for a function to be a two direction scaling function.

\section{Introduction}

Over the past two decades, Wavelet analysis, as a new development of mathematical analysis method, has been widely studied in both theoretics and applications. Now, wavelet analysis has been applied to the fields of signal analysis, image processing, medical imaging and diagnosis, differential equation and control theory.

American mathematician Daubechies [1] prove, in addition to Haar wavelet, other Unit-wavelet cannot all have compact support, symmetry and orthogonality. Recently, there have been done a lot of work in multi-scaling function and multiwavelet. Scale vector function with nonnegative mask has very important application in the engineering technology. Shouzhi Yang [2], introduces the concept of two-direction wavelet. As is known to all, refinement equation piays a very important role in wavelet theory and application [3-8], Two-directional refinement equation more general than fine equation also has a very important role in wavelet analysis.

Based on [8] this paper give two theorems in $L^{2}(R)$. From two-directional refinement equation, gave the concept of two-directional multi-resolution analysis and two-directional scaling function, discussed the some properties of two-directional scaling function, and finally gave a sufficient and necessary condition for a function to be a two-directional scaling function.

\section{Preliminary}

First, review the concept of two-directional refinement function and Parseval frame.

Definition 1. A function $\varphi(x)$ is said to be two-directional refinement function, if the following two-directional refinement equation is satisfied:

$$
\varphi(x)=\sum_{k} p_{k}^{+} \varphi(2 x-k)+\sum_{k} p_{k}^{-} \varphi(k-2 x)
$$

Where the sequence of $\left\{p_{k}^{+}\right\}_{k \in z}$ and $\left\{p_{k}^{-}\right\}_{k \in z}$ are respectively called forward two scale sequence and negative two scale sequence.

Definition 2. Let $H$ be a Hilbert space, $\left\{X_{j}\right\}_{j \in z} \subset H$, if there exist constants $c_{1}$ and $c_{2}$, $c_{1}, c_{2}>0$, such that

$$
c_{1}\|f\|^{2} \leq \sum_{j \in z}\left|<f, x_{j}>\right|^{2} \leq c_{2}\|f\|^{2}, \forall f \in H \text {; }
$$

Then, $\left\{x_{j}\right\}_{j \in z}$ is said to be the frame of $H$ space, if $c_{1}=c_{2},\left\{x_{j}\right\}_{j \in z}$ is said to be the tight frame of $H$ space, if $c_{1}=c_{2}=1,\left\{x_{j}\right\}_{j \in z}$ is said to be a Parseval frame of $H$ space. 
Now, we introduce two notions of operator and the basic notions and definitions used in the later paper.

Define translation operator as: $T_{k}: L^{2}(R) \rightarrow L^{2}(R), \quad f(\cdot) \mid \rightarrow f(\cdot-k), \forall f \in L^{2}(R), k \in Z$;

Define expansive operator as: $D_{j}: L^{2}(R) \rightarrow L^{2}(R), \quad f(\cdot) \mid \rightarrow 2^{\frac{j}{2}} f\left(2^{j} \cdot\right), \forall f \in L^{2}(R), \quad j \in Z$;

$L^{2}[0,2 \pi]$ denote the space of all $2 \pi$-periodic functions.

For all $f, g \in L^{2}(R)$, we denote by $[f, g]$ the $2 \pi$-periodic function

$$
[f, g](x)=\sum_{k \in z} f(x+2 k \pi) \overline{g(x+2 k \pi)} \text {. }
$$

It is easily seen that $[\because \cdot]$ is a sesquilinear form, $[f, g] \in L^{1}[0,2 \pi]$ for all $f, g \in L^{2}(R)$, and

$$
<f, g>=<f, g>=\int_{[0,2 \pi]}[\hat{f}, \hat{g}](\xi) d \xi .
$$

For $\sigma_{f}=[\hat{f}, \hat{f}]$, we let $\Omega_{f}$ be the $2 \pi$-translation invariant subset of $R$ defined, modulo a null set, by

$$
\Omega_{f}=\operatorname{supp} \sigma_{f}=\{\xi \in R: f(\xi+k) \neq 0, k \in Z\} .
$$

For a function $\varphi \in L^{2}(R)$ we denote by $\langle\varphi>$ the principal shift invariant space generated by $\varphi$; $\left\langle\varphi>\right.$ is the closed linear space generated by $T(\varphi)=\left\{T_{k} \varphi: k \in Z\right\}$.

\section{Two direction scaling function}

Before discussing two direction scaling function, we will introduce two theorem. The two theorems play an important role in the latter paper.

Theorem 1. Let $f \in L^{2}(R) \backslash\{0\}$.

(i) For an arbitrary $g \in L^{2}(R), k \in Z,<T_{k} f, g>$ is the kth Fourier coefficient of the function $[\hat{f}, \hat{g}] \in L^{1}[0,2 \pi]$.

(ii) $\langle f(\cdot), f(-\cdot)>\perp<g(\cdot), g(-\cdot)>$ if and only if $[\hat{f}(\cdot), \hat{g}(\cdot)]=0, \quad[\hat{f}(\cdot), \hat{g}(-\cdot)]=0$,

$$
[\hat{f}(-\cdot), \hat{g}(\cdot)]=0, \quad[\hat{f}(-\cdot), \hat{g}(-\cdot)]=0 .
$$

(iii) $\left\langle f(\cdot), f(-\cdot)>^{\wedge}=\left\{m_{1}(\cdot) \hat{f}(\cdot)+m_{2}(\cdot) \hat{f}(-\cdot), m_{1}, m_{2} \in L^{1}[0,2 \pi]\right\}\right.$.

(iv) $T(f(\cdot), f(-\cdot))=\left\{T_{k} f(\cdot), T_{l} f(-\cdot), k, l \in Z\right\}$ is a Parseval frame for $\langle f(\cdot), f(-\cdot)>$ if and only if

$$
[f, f](\xi)=\chi_{\Omega_{f}}(\xi),[\hat{f}, \hat{f}](-\xi)=\chi_{\Omega_{f}}(-\xi),[\hat{f}(-\xi), \hat{f}(\xi)]=[\hat{f}(\xi), \hat{f}(-\xi)]=0 .
$$

Prove. (i) since $\langle f, g\rangle=<\hat{f}, \hat{g}\rangle=\int_{[0,2 \pi]}[\hat{f}, \hat{g}](\xi) d \xi, \quad[m f, \hat{g}]=m[f, \hat{g}], m$ is $2 \pi$-periodic function, we have $\left.<T_{k} f, g\right\rangle=<\overline{e_{k}} \hat{f}, \hat{g}>=<[\hat{f}, \hat{g}], \overline{e_{k}}>_{L^{2}([0,2 \pi])}$.

(ii) follows immediately from (i).

(iii) Exactly as in the proof of Proposition 2.10(iii) in [8].

(iv) follows from (iii) we have $g=\left(m_{1}(\xi) \hat{f}(\xi)+m_{2}(\xi) \hat{f}(-\xi)\right)^{\vee} \in<f(\cdot), f(-\cdot)>$, By (i), we have $\sum_{k \in z} k g, T_{k} f(x)>\left.\right|^{2}+\sum_{l \in z} k g, T_{l} f(-x)>\left.\right|^{2}=\|[\hat{g}(\xi), \hat{f}(\xi)]\|_{L^{2}[0,2 \pi]}^{2}+\|[\hat{g}(\xi), \hat{f}(-\xi)]\|_{L^{2}[0,2 \pi]}^{2}$, since $\left\|m_{1}(\xi) \hat{f}(\xi)+m_{2}(\xi) \hat{f}(-\xi)\right\|^{2}=\sum_{k \in z}\left|<g, T_{k} f(x)>\right|^{2}+\sum_{l \in z}\left|<g, T_{l} f(-x)>\right|^{2}$. We obtain (iv) is true.

Theorem2. Let $\varphi(x) \in L^{2}(R), \varphi(-x) \in L^{2}(R), f_{i}(x) \in<\varphi(x), \varphi(-x)>, m_{i 1}(\xi) \in L^{2}([0,2 \pi])$ $m_{i 2}(\xi) \in L^{2}([0,2 \pi]), i=1,2$, such that $\hat{f}_{i}(2 \xi)=m_{i 1}(\xi) \hat{\varphi}(\xi)+m_{i 2}(\xi) \hat{\varphi}(-\xi)$, a.e. Then, $\left[f_{1}, f_{2}\right](2 \xi)$ $=m_{11}(\xi) \overline{m_{21}(\xi)} \sigma_{\varphi}(\xi)+m_{12}(\xi) \overline{m_{22}(\xi)} \sigma_{\varphi}(-\xi)+m_{11}(\xi+\pi) \overline{m_{21}(\xi+\pi)} \sigma_{\varphi}(\xi+\pi)$ $+m_{12}(\xi+\pi) \overline{m_{22}(\xi+\pi)} \sigma_{\varphi}(-\xi-\pi)$. 


\section{Proof.}

$\left.\left[\hat{f}_{1}, \hat{f}_{2}\right](2 \xi)=\sum_{k \in z} \hat{f}_{1}(2 \xi+2 k \pi) \overline{\hat{f}_{2}(2 \xi+2 k \pi)}=\sum_{k \in z} m_{11}(\xi+k \pi) \hat{\varphi}(\xi+k \pi)+m_{12}(\xi+k \pi) \hat{\varphi}(-\xi-k \pi)\right)$ $\left(\overline{m_{21}(\xi+k \pi) \hat{\varphi}(\xi+k \pi)+m_{22}(\xi+k \pi) \hat{\varphi}(-\xi-k \pi)}\right)$, We have (3) is true. We omit the details. Now, we introduce the notion of two direction MRA.

Definition 3. Let $\left\{V_{j}\right\}$ be a sequence of closed subspace of $L^{2}(R)$, where $V_{j}=$ close $_{L^{2}(R)}<2^{\frac{j}{2}} \varphi\left(2^{j} x-k\right), 2^{\frac{j}{2}} \varphi\left(l-2^{j} x\right), k, l \in Z>$, satisfying:

(i) $V_{j} \subset V_{j+1}, \forall j \in Z$;

(ii) $D V_{j}=V_{j+1}, \forall j \in Z$;

(iii) $\bigcap_{j \in z} V_{j}=\{0\}$;

(iv) $\overline{U_{j \in z} V_{j}}=L^{2}(R)$;

(v) There exists a function $\varphi \in V_{0}$ such that $\{\varphi(\cdot-k), \varphi(n-\cdot), k, n \in Z\}$ is a Parseval frame for $V_{0}$, We say $\left\{V_{j}\right\}$ is a two direction MRA for $L^{2}(R)$ and $\varphi$ is a two direction scaling function for $\left\{V_{j}\right\}$.

In order to get a necessary and sufficient condition for a function to be a two direction scaling function, we firstly discuss some propositions of twodirection scaling function .

Let $\varphi \in L^{2}(R)$ is a two direction scaling function for an $M R A\left\{V_{j}\right\}$. Consider the closed subspace $D^{j}<\varphi(\cdot), \varphi(-\cdot)>, j \in Z$, and take an arbitrary $f \in D^{j}\langle\varphi(\cdot), \varphi(-\cdot)>$, Then, by definition $f\left(2^{-j}\right) \in<\varphi(\cdot), \varphi(-\cdot)>$, by $1((\mathrm{iii})$-(iv)), we have

$$
D^{j}\left\langle\varphi(\cdot), \varphi(-\cdot)>=\left\{f \in L^{2}(R), f\left(2^{j} \xi\right)=\quad m_{1}(\xi) \hat{\varphi}(\xi)+m_{2}(\xi) \hat{\varphi}(-\xi), m \in L^{2}([0,2 \pi]), j \in Z\right\} .\right.
$$

Definition 4. For $f \in V_{1}$, we call the function $M_{f}=\left[\begin{array}{c}m_{f 1} \\ m_{f 2}\end{array}\right]^{T}$ a minimal filter for $f$, where $m_{f 1}=[f(2 \xi), \hat{\varphi}(\xi)], \quad m_{f 2}=[f(2 \xi), \hat{\varphi}(-\xi)]$, Since $\varphi \in V_{0} \subset V_{1}$, we have $\hat{\varphi}(2 \xi)=M_{\varphi}(\xi)\left[\begin{array}{c}\hat{\varphi}(\xi) \\ \hat{\varphi}(-\xi)\end{array}\right]$, (5). The function $M_{\varphi}$ will sometimes be called the minimal low-pass filter.

Proposition 5. Let $\left\{V_{j}\right\}$ be an MRA with a two direction scaling function $\varphi$ whose minimal filter is $M_{\varphi}$. Then

$$
\chi_{\Omega_{\varphi}}(2 \xi)=\left|m_{\varphi 1}(\xi)\right|^{2}+\left|m_{\varphi 2}(\xi)\right|^{2}+\left|m_{\varphi 1}(\xi+\pi)\right|^{2} \quad+\left|m_{\varphi 2}(\xi+\pi)\right|^{2} \quad \text { a.e. }
$$

$$
\begin{aligned}
&\text { Proof. } \left.[\hat{\varphi}, \hat{\varphi}](2 \xi)=\sum_{k \in I} \hat{\varphi}(2 \xi+2 k \pi) \overline{\hat{f}_{2}(2 \xi+2 k \pi)}\right) \\
&=\left|m_{\varphi 1}(\xi)\right|^{2} \sigma_{\varphi}(\xi)+\left|m_{\varphi 2}(\xi)\right|^{2} \sigma_{\varphi}(-\xi)+\left|m_{\varphi 1}(\xi+\pi)\right|^{2} \sigma_{\varphi}(\xi+\pi)+\left|m_{\varphi 2}(\xi+\pi)\right|^{2} \sigma_{\varphi}(-\xi-\pi) \text {. So }
\end{aligned}
$$
is true.

Proposition 6. Let $\left\{V_{j}\right\}$ be an MRA with a two direction scaling function $\varphi$. Then

$$
\lim _{j \rightarrow \infty}\left(\left|\hat{\varphi}\left(2^{-j} \xi\right)\right|^{2}+\left|\hat{\varphi}\left(-2^{-j} \xi\right)\right|^{2}\right)=1 \text { a.e. }
$$

Proof. we can show that (7) exactly as in the proof of Proposition 3.5 in [8], the only difference is that the system $D^{j} T(\varphi(\cdot), \varphi(-\cdot))$ is a Parseval frame for $D_{j} V_{0}$ for each $j \in Z$.

The following theorem is a necessary and sufficient condition for a function to be a two direction scaling function. 
Theorem 7. A function $\varphi \in L^{2}(R)$ is a scaling function for a $M R A$ if and only if the following three conditions are satisfied:

(i) $\hat{\varphi}(2 \xi)=m_{1}(\xi) \varphi(\xi)+m_{2}(\xi) \varphi(-\xi)$ for $m_{1}, m_{2} \in L^{2}[0,2 \pi]$.

(ii) $\lim _{j \rightarrow \infty}\left(\left|\hat{\varphi}\left(2^{-j} \xi\right)\right|^{2}+\left|\hat{\varphi}\left(-2^{-j} \xi\right)\right|^{2}\right)=1 \quad$ a.e.

(iii) $\sum_{k \in z}|\hat{\varphi}(\xi+2 k \pi)|^{2}=\chi_{\Omega_{\varphi}}(\xi), \quad$ a.e.

Proof. We already know that the above conditions are necessary ( cf.) (5), Propositions 6 and theorem 1(iv). Let us prove the converse.

Let us define $V_{0}=<\varphi(x), \varphi(-x)>$ and $V_{j}=D^{j} V_{0}, j \in Z$. Let $M_{\varphi}=\left[m_{1}(\xi), m_{2}(\xi)\right]$, by the proposition 5 , we have

$\chi_{\Omega_{\varphi}}(2 \xi)=\left|m_{1}(\xi)\right|^{2}+\left|m_{2}(\xi)\right|^{2}+\left|m_{1}(\xi+\pi)\right|^{2}+\left|m_{2}(\xi+\pi)\right|^{2}$ a.e.

For an arbitrary $f \in V_{0}$, by (4) and (5), we have

$f(2 \xi)=t_{1}(2 \xi) \hat{\varphi}(2 \xi)+t_{2}(2 \xi) \hat{\varphi}(-2 \xi),\left(t_{1}, t_{2} \in T[0,2 \pi]\right)$

$=\left[t_{1}(2 \xi) m_{1}(\xi)+t_{2}(2 \xi) m_{2}(-\xi)\right] \hat{\varphi}(\xi)+\left[t_{1}(2 \xi) m_{2}(\xi)+t_{2}(2 \xi) m_{1}(-\xi)\right] \hat{\varphi}(-\xi)$.

Then, $t_{1}(2 \xi) m_{1}(\xi)+t_{2}(2 \xi) m_{2}(-\xi), t_{1}(2 \xi) m_{2}(\xi)+t_{2}(2 \xi) m_{1}(-\xi), \in L^{2}[0,2 \pi]$.

Now (9) shows, using (4), that $f \in V_{1}$. This proves $V_{0} \subseteq V_{1}$, and then $V_{j} \subseteq V_{j+1}, \forall j \in Z \forall j \in Z$, is an immediate consequence.

Let us prove $\bigcap_{j \in z} V_{j}=\{0\}$.

We can show that $\bigcap_{j \in z} V_{j}=\{0\}$ exactly as in the proof of Theorem 5 in [2]. The only difference is that in our situation the system $\left\{\varphi_{0, k}^{+}(x), \varphi_{0, k}^{-}(x), k \in Z\right\}$ is a Parseval frame for $V_{0}$, but the reader can easily convince herself that the aforementioned argument from [2] survives.

Let us prove $\overline{U_{j \in z} V_{j}}=L^{2}(R)$. Let $f(x) \in\left(\bigcap_{j \in z} V_{j}\right)^{\perp}$, as demonstrated in [2, Theorem 6], Here we can use the same argument, we have $f=0$, this shows that $\overline{U_{j \in z} V_{j}}=L^{2}(R)$.

\section{Conclution}

In this paper, we follow the notion of $M R A$ and scaling function in $L^{2}(R)$, give the notions of two-directional MRA and two-directional scaling function, two properties in the subspace of $L^{2}(R)$ are generalized, we obtain two theorems, discuss some properties of two-directional scaling function, gave a sufficient and necessary condition for a function to be a two-directional scaling function.

\section{References}

[1] Daubechies I. Orthonormal basis of compactly supported wavelet [J]Comm Pure and Appl Math, 1988,41:909-996.

[2] Yang Shou zhi, Li You fa. two direction refinable function and two direction wavelet with high approximation order and regularity[J].:China series A-math: Math, 2007, 37(7): 779-795.

[3] Daubechies I, Lagarias J C. Two-scale difference equation I: existence and global regularity, of solutions[J]. SIAM Math Anal, 1991,22: 138 8-1 410.

[4] Daubechies I ,Lagarias J C. Two-scale difference equation II : Local regularity, infinite products of matrices and fractal[J]. SIAM Math Anal, 1992,23: 1 031-1 079.

[5] Dahmen W , Micchelli C. Biorthogonal wavelet expansions[J]. Constr Approx , 1977,13:293-328. 
[6] Song Li, Yang Jian bin. Vector refinement equationswith infinitely supportmasks[J].Journal of Approximation theory, 2007 , $148: 158-176$.

[7] Lawton W, Lee S L. Shen Z. Characterization of compactly supported refinable splines[J]. Adv Comput Math, 1995,3 :137-145.

[8] Bakic D, Krishtal I, Wilson EN. Parseval frame wavelets with $E_{n}^{(2)}$-dilations. Appl Comput. Harmon. Anal. 2005; 19(3):386431. 